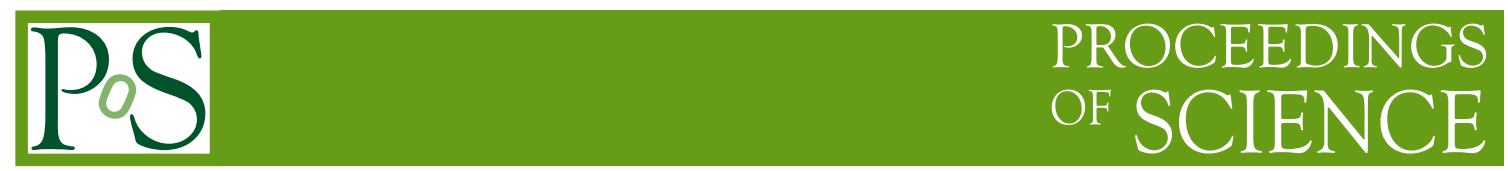

\title{
Interplanetary Coronal Mass Ejection and the Sun's Shadow Observed by the Tibet Air Shower Array
}

M. Amenomori, ${ }^{a}$ X. J. Bi, ${ }^{b}$ D. Chen, ${ }^{c}$ T. L. Chen, ${ }^{d}$ W. Y. Chen, ${ }^{b}$ S. W. Cui, ${ }^{e}$ Danzengluobu, ${ }^{d}$ L. K. Ding, ${ }^{b}$ C. F. Feng, ${ }^{f}$ Zhaoyang Feng, ${ }^{b}$ Z. Y. Feng, ${ }^{g}{ }^{g}$ Q. B. Gou, ${ }^{b}$ Y. Q. Guo, ${ }^{b}$ H. H. He, ${ }^{b}$ Z. T. He, ${ }^{e}$ K. Hibino, ${ }^{h}$ N. Hotta, ${ }^{i}$ Haibing Hu, ${ }^{d}$ H. B. Hu, ${ }^{b}$ J. Huang, ${ }^{b}$ H. Y. Jia, ${ }^{g}$ L. Jiang, ${ }^{b}$ F. Kajino, ${ }^{j}$ K. Kasahara, ${ }^{k}$ Y. Katayose, ${ }^{l}$ C. Kato, ${ }^{m}$ K. Kawata ${ }^{*}{ }^{n}$ M. Kozai, ${ }^{o m}$ Labaciren, ${ }^{d}$ G. M. Le, ${ }^{p}$ A. F. Li, ${ }^{q f b}$ H. J. Li, ${ }^{d}$ W. J. Li, ${ }^{b g}$ C. Liu, ${ }^{b}$ J. S. Liu, ${ }^{b}$ M. Y. Liu, ${ }^{d}$ H. Lu, ${ }^{b}$ X. R. Meng, ${ }^{d}$ T. Miyazaki ${ }^{m}{ }^{m}$ K. Mizutani, ${ }^{k r}$ K. Munakata, ${ }^{m}$ T. Nakajima, ${ }^{m}$ Y. Nakamura, ${ }^{m}$ H. Nanjo, ${ }^{a}$ M. Nishizawa, ${ }^{3}$ T. Niwa, ${ }^{m}$ M. Ohnishi, ${ }^{n}$ I. Ohta, ${ }^{t}$ S. Ozawa, ${ }^{k}$ X. L. Qian, ${ }^{f b}$ X. B. Qu, ${ }^{u}$ T. Saito, ${ }^{v}$ T. Y. Saito, ${ }^{w}$ M. Sakata, ${ }^{j}$ T. K. Sako, ${ }^{x n}$ J. Shao, ${ }^{b f}$ M. Shibata, ${ }^{l}$ A. Shiomi, ${ }^{y}$ T. Shirai, ${ }^{h}$ H. Sugimoto, ${ }^{z}$

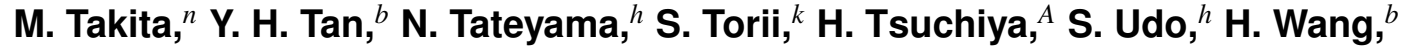
H. R. Wu, ${ }^{b}$ L. Xue,${ }^{f}$ Y. Yamamoto, ${ }^{j}$ K. Yamauchi, ${ }^{l}$ Z. Yang, ${ }^{b}$ A. F. Yuan, ${ }^{d}$ T. Yuda,$^{n}$ L. M. Zhai, ${ }^{c}$ H. M. Zhang, ${ }^{b}$ J. L. Zhang, ${ }^{b}$ X. Y. Zhang, ${ }^{f}$ Y. Zhang, ${ }^{b}$ Yi Zhang, ${ }^{b}$ Ying Zhang, ${ }^{b}$ Zhaxisangzhu $^{d}$ and X. X. Zhou ${ }^{g}$ (The Tibet AS $\gamma$ Collaboration) 
${ }^{a}$ Department of Physics, Hirosaki University, Japan

${ }^{b}$ Key Laboratory of Particle Astrophysics, Institute of High Energy Physics, Chinese Academy of Sciences, China

${ }^{c}$ National Astronomical Observatories, Chinese Academy of Sciences, China

${ }^{d}$ Department of Mathematics and Physics, Tibet University, China

${ }^{e}$ Department of Physics, Hebei Normal University, China

${ }^{f}$ Department of Physics, Shandong University, China

${ }^{g}$ Institute of Modern Physics, SouthWest Jiaotong University, China

${ }^{h}$ Faculty of Engineering, Kanagawa University, Japan

${ }^{i}$ Faculty of Education, Utsunomiya University, Japan

${ }^{j}$ Department of Physics, Konan University, Japan

${ }^{k}$ Research Institute for Science and Engineering, Waseda University, Japan

${ }^{l}$ Faculty of Engineering, Yokohama National University, Japan

${ }^{m}$ Department of Physics, Shinshu University, Japan

${ }^{n}$ Institute for Cosmic Ray Research, The University of Tokyo, Japan

${ }^{o}$ Institute of Space and Astronautical Science, Japan Aerospace Exploration Agency (ISAS/JAXA),

Japan

${ }^{p}$ National Center for Space Weather, China Meteorological Administration, China

${ }^{q}$ School of Information Science and Engineering, Shandong Agriculture University, China

${ }^{r}$ Saitama University, Japan

${ }^{s}$ National Institute of Informatics, Japan

${ }^{t}$ Sakushin Gakuin University, Japan

${ }^{u}$ College of Science, China University of Petroleum, China

${ }^{v}$ Tokyo Metropolitan College of Industrial Technology, Japan

${ }^{w}$ Max-Planck-Institut für Physik, Deutschland

${ }^{x}$ Escuela de Ciencias Físicas y Nanotechnología, Yachay Tech, Ecuador

${ }^{y}$ College of Industrial Technology, Nihon University, Japan

${ }^{z}$ Shonan Institute of Technology, Japan

A Japan Atomic Energy Agency, Japan

E-mail: kawatadicrr.u-tokyo.ac.jp

We continuously observed the Sun's shadow in $3 \mathrm{TeV}$ cosmic-ray intensity with the Tibet-III air shower array since 2000. We find a clear solar-cycle variation of the deficit intensity in the Sun's shadow during the periods between 2000 and 2009. The MC simulation of the Sun's shadow based on the coronal magnetic field model does not well reproduce the observed deficit intensity around the solar maximum. However, when we exclude the transit periods during ICMEs towards to the Earth, the MC simulation shows better reproducibility. In the present paper, we report on the MC simulation and the analysis method of the Sun's shadow observed by the Tibet-III array.

35th International Cosmic Ray Conference - ICRC2017

10-20 July, 2017

Bexco, Busan, Korea

\footnotetext{
*Speaker.

${ }^{\dagger}$ Deceased.
} 


\section{Introduction}

The interplanetary Coronal Mass Ejection (ICME) is a large magnetized plasma ejected from the solar corona sometimes accompanying a solar flare. An ICME propagating directly toward the Earth interacts with the geomagnetic field and causes a geomagnetic storm and an aurora. An interplanetary shock driven in front of a fast ICME accelerates the plasma particles in the solar wind. According to the satellite observations of ICMEs near the Earth, the magnetic field strength inside the ICME is stronger than that of outside, so-called the magnetic cloud [U]. The propagation and evolution of the magnetic clouds have been estimated using the Magneto-Hydro-Dynamic (MHD) simulation. This kind of numerical simulation is very important rule for the short and long-term space weather prediction.

The Sun with an optical diameter of about $0.5^{\circ}$ viewed from the Earth blocks very-high-energy cosmic rays coming from the direction of the Sun and casts a shadow in the cosmic-ray intensity, which is possibly influenced by the solar magnetic field [ [2]. The Tibet air shower array has been in operation at Yangbajing (4,300 m above sea level) in Tibet, China since 1990, observing very-highenergy cosmic rays in the multi-TeV energy region. The first small Tibet-I array, which consists of 45 scintillation detectors with a $15 \mathrm{~m}$ spacing, successfully observed the first evidence of the

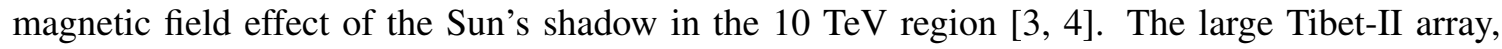
which consists of 221 scintillation detectors with a $15 \mathrm{~m}$ spacing, has been fully operated since 1996. With this array, a clear solar-cycle variation of the Sun's shadow is seen in the $10 \mathrm{TeV}$ during a full solar cycle from 1996 to 2009 [5]. In order to clarify the physical implications of the observed solar cycle variation, we develop numerical simulations of the Sun's shadow, using the Potential Field Source Surface (PFSS) model and the Current Sheet Source Surface (CSSS) model for the coronal magnetic field. We find that the deficit intensity in the simulated Sun's shadow is very sensitive to the coronal magnetic field structure, and the observed variation of the Sun's shadow is better reproduced by the CSSS model. This is the first successful attempt to evaluate the coronal magnetic field models by using the Sun's shadow observed in the $10 \mathrm{TeV}$ cosmic-ray flux.

The array was further upgraded to a higher density array (Tibet-III) with a $7.5 \mathrm{~m}$ spacing in late of 1999 to observe cosmic rays with lower energy threshold around a few TeV. In this proceedings, we analyze the Sun's shadow observed by the Tibet-III air shower array, and investigate the influence of the ICME on the Sun's shadow in the $3 \mathrm{TeV}$ energy region during periods between 2000 and 2009.

\section{Analysis}

We analyze the cosmic-ray events observed by the Tibet-III air-shower array from the direction of the Sun during period between 2000 and 2009. The overall angular resolution and the modal energy of the Tibet-III are estimated to be $0.9^{\circ}$ and $3 \mathrm{TeV}$, respectively. For the analysis of the Sun's shadow, the number of on-source events $\left(N_{\text {on }}\right)$ is defined as the number of events arriving from the direction within a circle of $0.9^{\circ}$ radius centered at the Sun. The number of background or off-source events $\left(\left\langle N_{\text {off }}\right\rangle\right)$ is then calculated by averaging the number of events within each of the eight off-source windows which are located at the same zenith angle as the on-source window, but 
apart by $\pm 6.4^{\circ}, \pm 9.6^{\circ}, \pm 12.8^{\circ}$ and $\pm 16.0^{\circ}$ in the azimuthal direction. We then estimate the flux deficit relative to the number of background events as $D_{\text {obs }}=\left(N_{\text {on }}-\left\langle N_{\text {off }}\right\rangle\right) /\left\langle N_{\text {off }}\right\rangle$.

\section{Sun's Shadow Simulation}

We have performed a detailed Monte Carlo (MC) simulation of the Sun's shadow. The antiparticles are traced back to the Sun from the Earth in the solar magnetic field models to reproduce the cosmic-ray shadow. For the magnetic field models and the MC simulation procedure are almost same as those used in the previous work [G]. We adopt the CSSS model, as a source surface (SS) model. The SS models describe the solar coronal magnetic field based on the observed photospheric magnetic field. In order to reproduce the temporal variation of the Sun's shadow, we use the photospheric magnetic field observed with the spectromagnetograph of the National Solar Observatory at Kitt Peak [ [ $]$ in each Carrington rotation (CR) period ( 27.3 days). The CSSS model [ [] includes the large-scale horizontal currents. This model involves four free parameters, $R_{\mathrm{ss}}, n$, the radius $R_{\mathrm{cp}}\left(<R_{\mathrm{ss}}\right)$ of the spherical surface where the magnetic cusp structure in the helmet streamers appears and the length scale of horizontal electric currents in the corona $l_{a}$. Here, we examine a standard value $R_{\mathrm{Ss}}=2.5 R_{\odot}$ used in the original paper [ $[\mathrm{d}]$. The other parameters, $n, R_{\mathrm{cp}}$ and $l_{a}$ are set to $10,1.7 R_{\odot}$ and $1.0 R_{\odot}$, respectively [ $[\mathrm{B}]$. For the interplanetary magnetic field (IMF) model and geomagnetic field, we adopt the Parker spiral model [Q] and a simple dipole model, respectively.

For the primary cosmic rays, we use the energy spectra and its elementary compositions modeled by Shibata et al. [ए]] to represent various measurements in the energy range of $0.3 \mathrm{TeV}$ to $1000 \mathrm{TeV}$. Air shower events are generated at the top of the atmosphere along the Sun's orbit around the Earth, using the CORSIKA code [W] with EPOS-LHC [ए2] interaction model. The air shower core of each simulated event is uniformly distributed over a circular region with a $300 \mathrm{~m}$ radius centered at the array. In order to treat the MC events in the same way as the events in the experimental data, these simulated events are distributed among the detectors in the same detector configuration as in the Tibet-III array by the GEANT4 code [[13]]. After air shower reconstruction and event selections, we assign the opposite charge to the remaining primary particles. These antiparticles are randomly shot back toward directions, each called the initial shooting direction. The particle track influenced by the solar magnetic fields between the Earth and the Sun is calculated by the fourth-order Runge-Kutta. Suppose the primary particle hits the Sun, its initial shooting direction should be equivalent to the observed particle direction shielded by the Sun. Finally, these initial shooting directions are smeared by the angular resolution event by event. In this way, the expected Sun's shadow becomes equivalent to the observed Sun's shadow.

\section{Results and Discussions}

In order to investigate the influence of the ICMEs on the Sun's shadow, we use a catalog of ICMEs recorded near the Earth [U4]]. This catalog includes 228 ICMEs during the analysis years between 2000 and 2009. While an ICME arrives at the Earth $\sim 4$ days after leaving the Sun, a cosmic ray takes only $\sim 8$ minutes to reach the Earth. Therefore, cosmic rays which arrive at the Earth after passing near the Sun are possibly affected by the ICME during a transit period between 


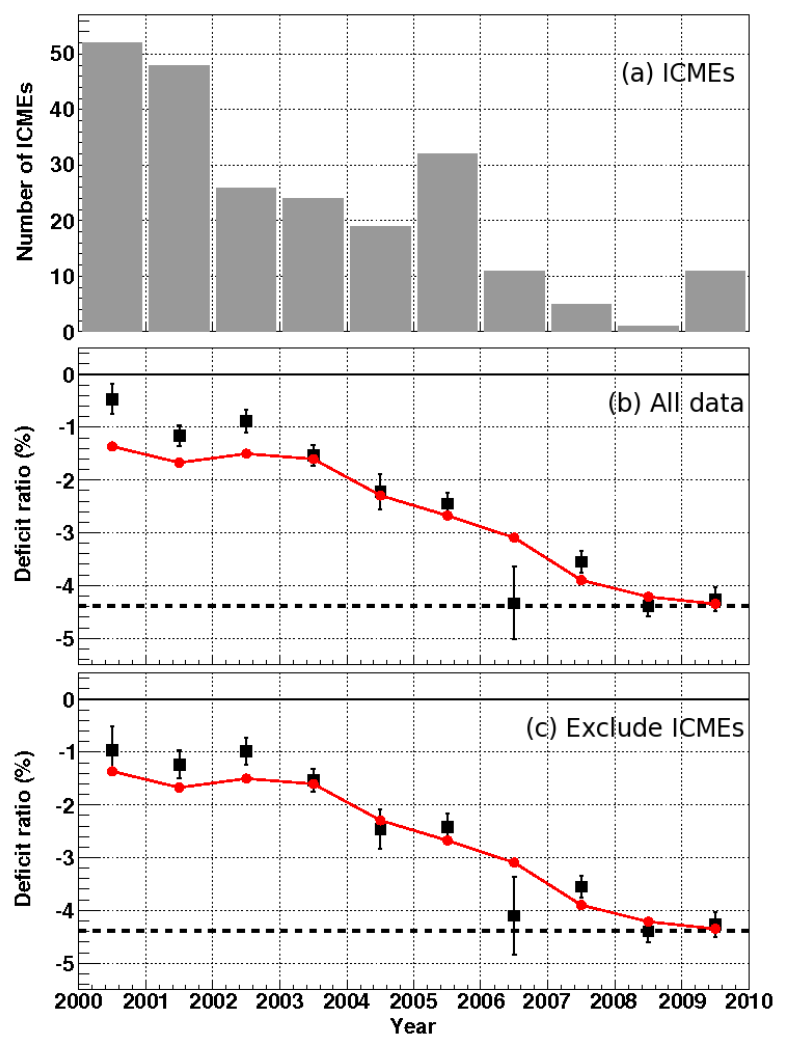

Figure 1: Temporal variation of (a) number of ICMEs recorded in each year in the catalog [प14], (b) the deficit intensity of the Sun's shadow $\left(D_{\mathrm{obs}}\right)$ in the $3 \mathrm{TeV}$ cosmic-ray intensity observed during an entire period, (c) $D_{\text {obs }}$ observed during a period without the ICME transit periods. The dashed lines in panels (b) and (c) indicate deficits expected from the apparent angular size of the Sun. The red circles indicate the central deficits $\left(\mathrm{D}_{\mathrm{MC}}\right)$ predicted by the MC simulation assuming the CSSS model.

the eruption observed by the SOHO/LASCO on the solar surface and the arrival at the Earth. In this study, we analyze the data excluding the transit periods of all ICMEs listed in the Richardson \& Cane catalog and examine whether the influence of ICMEs on the Sun's shadow. For ICMEs lacking relevant observation by the SOHO/LASCO, we assume the transit time to be 4 days, which is an average of all ICMEs with the SOHO/LASCO observation.

Figure $\square$ (a) shows the number of ICMEs per year which looks roughly proportional to the solar activity. The histogram shows all 228 ICMEs listed in the ICME catalog. While a half of all analysis period is occupied by the ICME transit periods in 2000-2002 around the solar maximum, $>90 \%$ of all analysis period is left without the ICME transit period in 2007-2009 around the solar minimum due to the low ICME rate. Figure $\mathbb{W}(\mathrm{b})$ and (c) show the deficit intensity variations of the Sun's shadow using all the data (including ICME periods), and the data after excluding ICME periods, respectively. The black squares and red circles in Figure $\square$ show the observed deficit intensity variations and those predicted by the MC simulation, respectively. Clearly seen in Figure $\square$ (b) is the deficit intensity in the Sun's shadow varying in a correlation with the solar activity. The deficit intensity by the MC simulation significantly overestimates the observed one around the solar maximum. However, when the transit periods of ICMEs listed in the ICME catalog are 
excluded from the data, the MC simulation with the CSSS model better reproduces the observation. This result implies that the magnetic fields in the ICMEs moving toward the Earth from the Sun affect the cosmic ray orbits across the ICMEs. The MHD simulations including ICMEs have been developed to reproduce the propagation and evolution in the IMF which is an essential factor for the space weather prediction [대, [0]]. In the future, we will investigate $\mathrm{TeV}$ cosmic-ray behavior in the magnetic cloud as the ICME by incorporating these kinds of MHD simulations with our MC simulation of the Sun's shadow.

\section{Conclusion}

We confirmed a clear solar-cycle variation of the Sun's shadow in the $3 \mathrm{TeV}$ cosmic rays observed by the Tibet-III air shower array during a period between 2000 and 2009. The temporal variation of the deficit intensity is compared with the MC simulation assuming the CSSS model for the coronal magnetic field. The MC simulation significantly overestimates the intensity deficit in the Sun's shadow observed around the solar maximum. However, when the ICME periods in the catalog prepared by Richardson and Cane are excluded from the analysis, the MC simulation with the CSSS model better reproduces the observation. This result implies that the magnetic field in the ICME propagating toward the Earth from the Sun affects orbits of $\mathrm{TeV}$ cosmic rays across the ICMEs.

\section{Acknowledgments}

The collaborative experiment of the Tibet Air Shower Arrays has been performed under the auspices of the Ministry of Science and Technology of China (No. 2016YFE0125500) and the Ministry of Foreign Affairs of Japan. This work was supported in part by a Grant-in-Aid for Scientific Research on Priority Areas from the Ministry of Education, Culture, Sports, Science and Technology, by Grants-in-Aid for Science Research from the Japan Society for the Promotion of Science in Japan. This work is supported by the National Natural Science Foundation of China (Nos. 11533007 and 11673041) and the Chinese Academy of Sciences and the Key Laboratory of Particle Astrophysics, Institute of High Energy Physics, CAS. This work is supported by the joint research program of the Institute for Cosmic Ray Research (ICRR), The University of Tokyo. K. Kawata is supported by the Toray Science Foundation. The authors thank Dr. Xuepu Zhao of Stanford University for providing the usage of the CSSS model and Dr. K. Hakamada of Chubu University and Dr. Shiota of Nagoya University for supplying the data calculated by the Potential Field Source Surface (PFSS) model. The authors thank Dr. József Kóta of University of Arizona for his useful comments and discussions.

\section{References}

[1] L. Burlaga, E. Sittler, F. Mariani and R. Schwenn, Magnetic loop behind an interplanetary shock: Voyager, Helios, and IMP 8 observations, J. of Geophys. Res., 86, 6673 (1981).

[2] G. W. Clark, Arrival Directions of Cosmic-Ray Air Showers from the Northern Sky, Phys. Rev., 108, 450 (1957). 
[3] M. Amenomori, et al., Direct Evidence of the Interplanetary Magnetic Field Effect on the Cosmic-Ray Shadow by the Sun, ApJ, 415, L147 (1993).

[4] M. Amenomori, et al., Shadowing of Cosmic Rays by the Sun near Maximum or at the Declining Phase of Solar Activity, ApJ, 464, 954 (1996).

[5] M. Amenomori, et al., Probe of the Solar Magnetic Field Using the "Cosmic-Ray Shadow" of the Sun, PRL, 111, 011101 (2013).

[6] H. P. Jones et al., The NASA/NSO Spectromagnetograph, Solar Phys., 139, 211 (1992).

[7] X. P. Zhao and J. T. Hoeksema, Prediction of the interplanetary magnetic field strength, J. of Geophys. Res.. 100, 19 (1995).

[8] M. Schüssler and I. Baumann, Modeling the Sun's open magnetic flux, Astron. and Astrophys., 459, 945 (2006).

[9] E. N. Parker, Dynamics of the Interplanetary Gas and Magnetic Fields, ApJ, 128, 664 (1958).

[10] M. Shibata, Y. Katayose, J. Huang and D. Chen, Chemical Composition and Maximum Energy of Galactic Cosmic Rays, ApJ, 716, 1076 (2010).

[11] D. Heck, J. Knapp, J. N. Capdevielle, G. Shatz and T. Thouw, CORSIKA: A Monte Carlo Code to Simulate Extensive Air Showers, (FZKA 6019)(Karlsruhe: Forschungszentrum Karlsruhe (1998).

[12] T. Pierog, Iu. Karpenko, J. M. Katzy, E. Yatsenko and K. Werner, EPOS LHC: Test of collective hadronization with data measured at the CERN Large Hadron Collider, Phys. Rev. C, 92, 034906 (2015).

[13] S. Agostinelli, et al., GEANT4-a simulation toolkit, Nucl. Instrum. Methods Phys. Res. A, 506, 250 (2003).

[14] I. G. Richardson and H. V. Cane, Near-Earth Interplanetary Coronal Mass Ejections During Solar Cycle 23 (1996-2009): Catalog and Summary of Properties, Solar Phys., 264, 189 (2010).

[15] D. Shiota, K. Kusano, T. Miyoshi and K. Shibata, Magnetohydrodynamic Modeling For A Formation Process of Coronal Mass Ejections: Interaction Between An Ejecting Flux and An Ambient Field, ApJ, 718, 1305 (2010).

[16] M. Den, T. Tanaka, Y. Kubo and S. Watari, Three-dimensional MHD simulation of the solar wind from the solar surface to 400 solar radius using REPPU (REProduce Plasma Universe) code, in proceedings of the 34th International Cosmic Ray Conference, POS (ICRC2O15) 184 (2015). 